\title{
Un videojuego que promueve la participación ciudadana interactiva en las reformas urbanas
}

Germán David Bello-Maldonado

Universidad de los Andes

arq.davidbello@gmail.com

DOI: https://doi.org/10.21158/2357514x.v7.n2.2019.2573

Cómo citar este artículo: Bello-Maldonado, G. D. (2019). Un videojuego que promueve la participación ciudadana interactiva en las reformas urbanas. Revista Virtu@lmente, 7(2), 95-112. DOI: https://doi.org/10.21158/2357514x.v7.n2.2019.2573

Fecha de recepción: 22 de abril 2019

Fecha de aprobación: 03 de junio de 2019

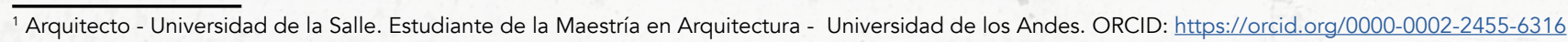




\section{RESUMEN}

Este artículo describe el proceso de creación de City Co, un videojuego que tiene como objetivo concientizar a la población joven sobre la relevancia y la implicación del desarrollo territorial. Para exponer el contexto y la relevancia del videojuego, primero se presenta una descripción de la planificación urbana y su relación con la vida de cada ciudadano, luego una muestra de algunos desafíos en el urbanismo, los beneficios de la participación ciudadana y el uso de medios tecnológicos en el desarrollo territorial. También se nombran algunos videojuegos city builders y algunos de sus aportes al pensamiento urbano. En el texto, se presentan y analizan las diferentes fases en la estructuración del prototipo de City Co, que en su prueba piloto toma como base el Plan Parcial El Triángulo de la ciudad de Bogotá, Colombia. Esto, para finalmente demostrar y concluir que los medios tecnológicos contribuyen a la información y a la comunicación del desarrollo territorial en las reformas urbanas y aportan a la generación de interés en el conocimiento y el entendimiento de procesos de planes urbanísticos. Por esta razón se puede afirmar que un videojuego como City Co, al igual que otros city builders, resulta pertinente en tanto que informa de modo significativo a la población joven y promueve en esta inquietudes sobre la planificación territorial

Palabras clave: gamificación urbana; participación ciudadana; city builders; urbanismo participativo; reformas urbanas; Plan Parcial El Triángulo. 


\section{A video game that promotes interactive citizen participation in urban reforms}

\section{ABSTRACT}

This article describes the creation process of City $\mathrm{Co}$, a video game that aims at raising awareness among the young population about the relevance and implication of territorial development. In order to expose the context and importance of the video game, we first present a description of urban planning and its relationship with each citizen's life, together with a sample of some challenges in urban planning, the benefits of citizen participation, and the use of technological means in territorial development, including the names of some city building video games and some of their contributions to urban thinking. In the text, we also present and analyze the different phases in the structuring of the City Co prototype, whose pilot test is based on the partial plan El Triángulo from the city of Bogotá, Colombia. This, to finally demonstrate and conclude that the technological media contribute to the information and communication of territorial development in urban reforms, and to the generation of interest in knowledge and understanding of urban planning processes. For this reason, it can be said that a video game like City Co, as well as other city building video games, is relevant in the sense that it significantly informs and promotes concerns in the young population about territorial planning.

Keywords: urban gamification; citizen participation; city builders; participatory urbanism; urban reforms; El Triángulo Partial Plan. 


\section{Um videogame que promove a participação cidadã interativa nas reformas urbanas}

\section{RESUMO}

Este artigo descreve o processo de criação de City Co, um videogame que tem como objetivo conscientizar a população jovem sobre a relevância e a implicação do desenvolvimento territorial. Para expor o contexto e a relevância do videogame, primeiro apresenta-se uma descrição do planejamento urbano e sua relação com a vida de cada cidadão. Em seguida, apresenta-se uma mostra de alguns desafios do urbanismo, os benefícios da participação cidadã e o uso de meios tecnológicos no desenvolvimento territorial. Também se indicam alguns videogames city builders e algumas de suas contribuições ao pensamento urbano. Apresentam-se e analisam-se as diferentes fases da estruturação do protótipo de City Co, que em sua prova piloto toma como base o Plan Parcial El Triángulo da cidade de Bogotá, Colômbia. Finalmente se demonstra e conclui que os meios tecnológicos contribuem com a informação e a comunicação do desenvolvimento territorial nas reformas urbanas e com a geração de interesse no conhecimento e no entendimento de processos de planejamentos urbanísticos. Por esta razão se pode afirmar que um videogame como City Co, assim como outros city builders, é apropriado para passar informações de modo significativo à população jovem e promover nela inquietações sobre o planejamento territorial.

Palavras-chave: gamificação urbana; participação cidadã; city builders; urbanismo participativo; reformas urbanas; Plan Parcial El Triángulo. 


\section{Conception d'un jeu vidéo participatif et interactif sur le thème de l'urbanisme}

\section{RÉSUMÉ}

Cet article décrit le processus de création de City Co, un jeu vidéo visant à sensibiliser un public jeune à la pertinence de l'implication dans le développement urbanistique et territorial. Pour exposer le contexte et la pertinence de ce jeu vidéo, nous proposerons d'abord une description de l'urbanisme et sa relation dans la vie de chacun puis nous présenterons certains des grands défis urbanistiques actuels, les avantages de la participation citoyenne et l'utilisation de la technologie dans le développement territorial. Différents concepteurs de jeux vidéo ainsi que leurs contributions à la pensée urbanistique seront également présentés. Nous analyserons par ailleurs les différentes phases de conception et de structuration du prototype de City Co et de son test pilote basé sur le Plan Partiel El Triángulo de la ville de Bogotá, Colombie. Nous insisterons pour conclure sur la pertinence de l'utilisation des moyens technologiques pour le développement territorial et les réformes urbaines afin de susciter l'intérêt pour la connaissance et la compréhension des processus d'urbanisme car nous avons la conviction que les jeux vidéo de type City Co permettent d'éduquer significativement les plus jeunes au concept en sucitant leur intérêt pour l'urbanisme et l'aménagement des territoires.

Mots-clés: jeux sérieux d'urbanisme; participation citoyenne; constructeurs urbains; planification urbaine participative; réformes urbaines; Plan Partiel El Triangulo. 


\section{Introducción}

En la Constitución Política de Colombia, artículo primero, encontramos que se considera el país un «Estado social de derecho organizado en forma de República unitaria, democrática, participativa, pluralista». Así mismo, en la Ley 388 de 1997 se encuentran directrices normativas que buscan gestionar el territorio junto con las múltiples implicaciones que esta labor conlleva. De igual modo, el Ministerio de Tecnologías de la Información y la Comunicación (MinTIC) — en su misión "promueve el acceso, uso efectivo y apropiación masivos de las Tecnologías de la Información y la Comunicación». La unión de estos tres elementos - democracia participativa, norma urbana y el uso efectivo de tecnologías - son las directrices con las que nace la idea de crear un medio que incentive la participación urbana en la planeación territorial con el uso de tecnologías que generen interés en la ciudadanía.

Es entonces que se plantea crear e implementar el prototipo de un videojuego que promueva la participación ciudadana interactiva en reformas urbanas, usando como prueba piloto el Plan Parcial El Triángulo, ubicado en Bogotá, Colombia.

Figura 1. Logo del videojuego City Co

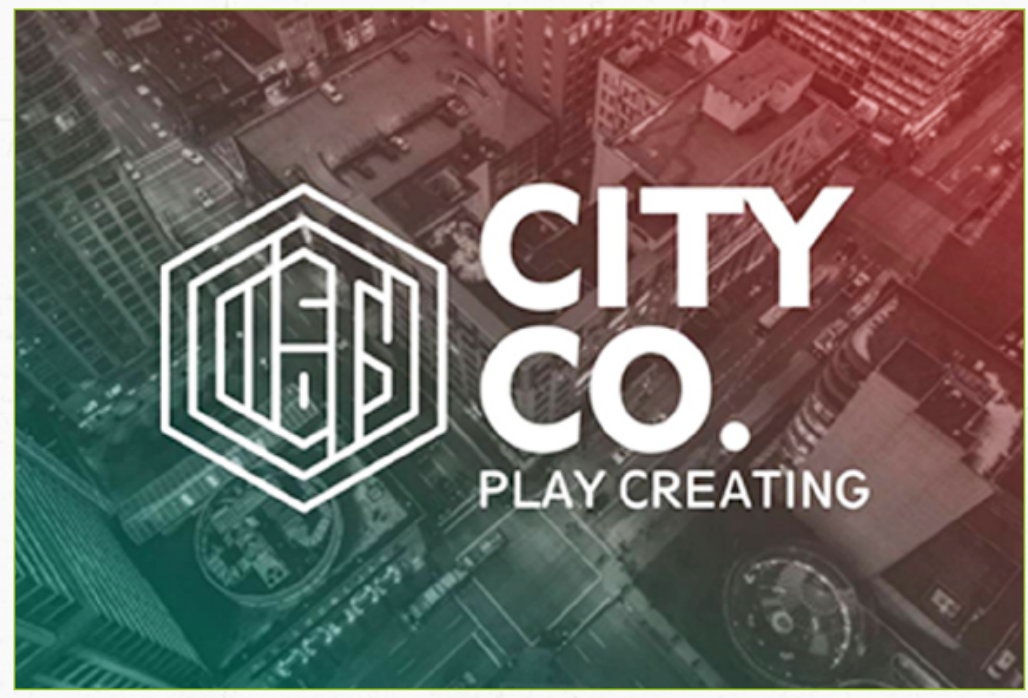

Fuente. Elaboración Cristian Maldonado. 


\section{Planificación urbana y su implicación en la vida de los ciudadanos}

Según Burden (2014), «las ciudades tratan fundamentalmente sobre las personas». No se trata de desconocer que las ciudades surgen por motivos humanos, en la búsqueda de suplir de un mejor modo, más sencillo y sofisticado las necesidades que nos atañen, desde las necesidades básicas que cuidan de la vida y la salud —como, por ejemplo, la alimentación, los medios o las redes para depositar los residuos orgánicos humanos, los sitios para vivir y compartir en sociedad-, hasta la experimentación con nuevas tecnologías orientadas a mejorar en distintas y diversas áreas en busca del confort y el progreso humano.

La planificación urbana es una labor bella pero desafiante. Teóricos en distintos momentos han discutido sobre el ideal de ciudad y, junto con esto, han buscado distintas formas de vivir; desde la Antigüedad, poseer un terreno ha sido de gran valor y ha sido sinónimo de poder y virtud, pero aún mayor relevancia ha tenido la capacidad de una adecuada administración de ese territorio.

Ahora bien, la planificación urbana nos compete a todos, bien sea de modo directo o indirecto, de manera que influencia nuestras vidas, los recursos que consumimos, el modo y el lugar en el que vivimos, nuestra cultura y apariencia. De igual forma, nos proporciona las opciones de medios y los tiempos que tendremos para transportarnos, trata del modo en que comercializamos y nos relacionamos, de los lugares a los que se nos permite ir, a los que no y a los que podemos acceder con restricciones; también, podríamos decir que la planeación territorial trata sobre la planeación proxémica ${ }^{2}$ de nuestra cotidianidad -en el prototipo de City Co esto se buscó articular a modo de esquema al dar a los jugadores la libertad, bajo parámetros establecidos ${ }^{3}$, de implementar las distancias que considerasen pertinentes en algunas edificaciones urbanas-. El planteamiento urbano es una insinuación hacia la cotidianidad de las relaciones interpersonales, ya que, si bien no se somete la voluntad de los ciudadanos en la labor urbana, arquitectónica y de planeación territorial, sí es posible proponer diferentes posibilidades de habitar el espacio.

Por su parte, Colomina menciona que «la arquitectura está basada en la idea del genio único cuando en realidad es colaboración; como el cine, todos deberían salir en los créditos» (Kochen, 2014, p.1). Desde el diseño de la estructura general de una metrópolis hasta la percepción de cada individuo que la habite y la viva, cada aporte cuenta. Así, este deseo de lograr mayor participación ciudadana en la planeación territorial y de buscar la concientización de las personas sobre la importancia en el desarrollo urbano es uno de los motivos de la creación del videojuego City Co.

${ }^{2}$ El antropólogo estadounidense Eduard Hall, en su libro The hidden dimension, relata la proxemia -lo que comunicamos con el espacio entre nosotros-. Habla, primero, sobre el comportamiento animal, y luego sobre el de las personas. Hall divide la proxemia en cuatro áreas: la íntima, la personal, la social y la de zonas públicas. Otros teóricos urbanos como, por ejemplo, Jan Gehl (2011, 2014), Jane Jacobs (2013) o Amanda Burden (2014), se han basado en aspectos de ese estudio antropológico para sustentar sus propias teorías urbanas.

${ }^{3}$ Esto, al tener en cuenta como guía algunas de las lecciones dadas por Jan Bazant en Manual de criterios urbanos (1984), así como parámetros establecidos por el Plan Parcial El Triángulo de Bogotá. 
En esta primera sección se ha descrito, de manera breve, la influencia de la planificación territorial en la vida de cada ciudadano. A continuación, se nombran desafíos que, para la particularidad de Bogotá, así como de otras ciudades, se presentan en temas de planeación territorial.

\section{Problemáticas urbanas que afectan a los ciudadanos}

Al ser el desarrollo urbano desafiante en razón a las múltiples variables que posee, surgen retos causados por la creciente demografía ${ }^{4}$, las múltiples necesidades sociales, las transformaciones medioambientales, la reorganización social y los modos en que se percibe un espacio.

Algunos ejemplos de los desafíos urbanos a los que nos enfrentamos son: la inclusión ciudadana para personas con algún tipo de discapacidad física, las problemáticas medioambientales, el diseño del espacio público y el diseño de vivienda. Algunas referencias al respecto se describen a continuación.

\subsection{Inclusión}

En Colombia, para el 2015, el Departamento Administrativo Nacional de Estadística reportó un 6,3 \% de la población con capacidades motrices alternativas, lo que equivale a 2 624898 personas (Ministerio de Salud y Protección Social, 2018). Ahora bien, en temas de reformas urbanas llega a ser pertinente que cada una de ellas conlleve diseños inclusivos para este tipo de población. Lamentablemente, es muy sencillo salir a muchas de las calles de Bogotá y observar andenes que dificultan la movilidad, bien sea para personas en silla de ruedas o mujeres con los coches de sus bebés, de modo que prefieren desplazarse sobre la acera vehicular, lo cual genera un conflicto entre peatones y vehículos automotrices. Como esta se encuentran otras problemáticas semejantes debido a deficiencias en la planificación.

\subsection{Medio ambiente}

De acuerdo con el artículo 79 de la Constitución Política de Colombia (1991),

todas las personas tienen derecho a gozar de un ambiente sano. La ley garantizará la participación de la comunidad en las decisiones que puedan afectarlo. Es deber del Estado proteger la diversidad e integridad del ambiente, conservar las áreas de especial importancia ecológica y fomentar la educación para el logro de estos fines.

${ }^{4}$ El secretario general de la ONU, Ban Ki-moon, habló el 17 de septiembre del 2014 sobre la importancia de una buena planificación de los entornos urbanos a fin de impulsar el desarrollo: «El titular de la ONU resaltó que la mitad de la población vive en centros urbanos y que a mediados de este siglo se prevé que más de dos tercios de la humanidad residirá en ciudades. Casi la mitad de ese crecimiento se concentrará en países en desarrollo. "La urbanización nos afecta a todos", subrayó Ban y agregó que las áreas urbanas están en el núcleo de muchos retos, oportunidades y promesas». 
En octubre del 2018, la revista Semana publicó que, en Bogotá, en lo recorrido de ese mismo año, se registró una concetranción promedio de las partículas PM10 de $38 \mathrm{ug} / \mathrm{m}^{3}$ -microgramo/metro cúbico-, lo cual equivale a casi dos veces el límite máximo recomendado por la Organización Mundial de la Salud, $20 \mathrm{ug} / \mathrm{m}^{3}$. En consencuencia, se deben implementar estrategias en búsqueda de soluciones para fenómenos como este.

\subsection{El espacio público}

Según Gehl ${ }^{5}$, la ciudad debe ser para la gente, pensando en su bienestar tanto individual como colectivo. Si bien el espacio público ha sido organizado con miras al disfrute social, también es testigo de las problemáticas que cotidianamente surgen, pues se sabe que al hablar de espacio público no solo se trata de una plazoleta, de una banca en medio de un prado, de unas escaleras que conectan el espacio privado con el exterior y un sinnúmero de cualidades más, sino de posibles relaciones interpersonales.

A mediados del 2017 Bogotá reportó un déficit del 74 \% de espacio público (Vega, 2017) en comparación con el requerido por la OMS, según el informe de la Administración Distrital, lo cual enfatiza el requerimiento de una mejor planeación en cuanto al espacio público. Al hablar de espacio público tratamos temas de interconectividad entre ciudadanos y el bienestar de estos.

\subsection{Vivienda}

Escallón (2014) 6 señala que la vivienda no es solo un tema que se deba mirar hacia el interior de la edificación, sino que esta se ve altamente influenciada por su contexto inmediato y de ciudad.

La unidad de inteligencia de la revista The Economist, en su ranquin de ciudades habitables para vivir, ubica a Bogotá en el puesto 108, con lo cual localiza a la capital colombiana entre las cuatro últimas posiciones de Latinoamérica ${ }^{7}$.

Los anteriores son temas que, de forma constante, en cada puesta a prueba y ponencia dada sobre City Co se han nombrado, recalcando las afectaciones que tenemos como individuos debido a la planificación territorial y la importancia de nuevas visiones que aporten a la resolución en torno a estos temas. Con esto también se busca incentivar la participación ciudadana en la planeación urbana.

\footnotetext{
${ }^{5}$ En su libro La humanización del espacio urbano Jan Gehl nombra múltiples cualidades del espacio público y su influencia en nuestra cotidianidad.

${ }^{6}$ La arquitecta Clemencia Escallón, profesora de la Universidad de los Andes con profundo interés en el tema de la vivienda y la ciudad, nombra la correspondencia que existe entre ambas al referir cómo una buena vivienda siempre será asociada a un buen entorno planificado de la ciudad.
}

7 La revista Semana, el 16 de agosto del 2018, publica una reseña al respecto con el título de su noticia: «Bogotá, cuarta entre las peores ciudades para vivir en América Latina». 


\section{Participación ciudadana y la era tecnológica en la planeación urbana}

De acuerdo con Rojas (2017),

\begin{abstract}
el conformismo y el desentendimiento de la ciudadanía frente a la gestión urbana es un factor que influye negativamente en la construcción de ciudad, ya que los profesionales y expertos en el tema requieren de la validez de la información en la toma de decisiones, pero esto no es solo un problema de desinformación, sino también de la limitación existente con las herramientas que se tienen para la participación de la ciudadanía y los múltiples actores que convergen en el territorio.
\end{abstract}

El director de planeación, Luis Fernando Mejía periodo 2014-2018-, habló ${ }^{8}$ con respecto a la participación ciudadana en torno al desarrollo urbano, todo lo cual son puntos que aportan a la regulación, así como a la información ciudadana, y que, finalmente, contribuyen a mejoras en la planificación territorial.

Algunas aplicaciones existentes que promueven la participación ciudadana con relación a aspectos urbanos son:

- Cityzn app. Permite realizar presupuestos participativos, propuestas ciudadanas o declarar incidentes en la ciudad.
- YoVeoVeo-Ecuador-. Consiste en la denuncia de los ciudadanos de incidentes que se dan en su entorno, bien sea en temas de salud, educación y transporte, o bien con respecto a obras públicas.

- Arrels Localizador-España- Es una aplicación de la Fundación Arrels que busca apoyar a los equipos que asisten a las personas sin hogar (Martisi, 20 de feberero de 2019).

En cuanto a insumos digitales usados como aporte a la planificación urbana y su entendimiento para Bogotá, se encuentran los siguientes: Google Maps', Google Earth, Ideca $^{10}$, Mapas Bogotá11, Sinupot ${ }^{12}$, IGAC $^{13}$, o CAR ${ }^{14}$. Estos son ejemplo de algunas de las aplicaciones o plataformas que encontramos y aportan cada una en su respectiva especificidad, bien sea que sirvian como visores o bien porque permiten observar proyectos futuros, normativa urbana, afectaciones viales y medioambientales, distribuciones urbanas tales como los usos en la ciudad, las alturas, los aislamientos o los respectivos planos de llenos y vacíos, solo por nombrar unos ejemplos de sus funcionalidades.

\footnotetext{
8 Ponencia presentada en el marco de la temática de desarrollo urbano y participación ciudadana, en la Universidad de los Andes en el 2018. ${ }^{9}$ Ofrece imágenes de mapas desplazables y fotografías por satélite.

${ }^{10}$ En su página principal encontramos que es el encargado de poner la información de Bogotá en mapas a fin de llevarlos a su gente.

11 Visualiza, consulta y descarga datos oficiales de Bogotá D. C.

${ }^{12}$ Sistema de Norma Urbana y Plan de Ordenamiento Territorial. Compila los componentes urbano y rural del Plan de Ordenamiento Territorial.

${ }^{13}$ El Instituto Geográfico Agustín Codazzi es la entidad encargada de producir el mapa oficial y la cartografía básica de Colombia.

${ }^{14}$ Corporaciones Autónomas Regionales y de Desarrollo Sostenible en Colombia son la primera autoridad ambiental a nivel regional.
} 


\section{Videojuegos city builders}

Seleccionados como aporte en razón al interés de concientizar a la población joven sobre la importancia de la planificación territorial, la cual nos afecta a todos, algunos de los ejemplos de videojuegos que destacamos por su aporte en la construcción de ciudades son: Sim city ${ }^{15}$, Anno $1800^{16}$, Flotsam ${ }^{17}$, Block'Hood ${ }^{18}$, Dawn of man $^{19}$, Buoyancy, Tropico 620, Planet coaster ${ }^{21}$, Atomic society ${ }^{22}$, Surviving Mars $^{23}$, FrostPunk ${ }^{24}$, y Cities skylines ${ }^{25}$.

Así, estos videojuegos abordan una diversidad de temas que van desde la historia hasta proyecciones de supervivencia en entornos externos a la Tierra, ponen a consideración de los jugadores recursos medioambientales, presupuestos y políticas, así como los colocan en el papel del dirigente y del planificador supremo. Incluso, en algunos observan las consecuencias de sus decisiones y la afectación a la población o se batalla por el desarrollo de la ciudad creada, y en otros por la supervivencia. De esta manera, cada uno desarrolla un área diferente de interés en torno al desarrollo urbano.

Después de este recuento de la motivación del videojuego City Co y su interés por concientizar sobre las reformas urbanas ${ }^{26}$, así como de proporcionar una muestra de algunos de los medios digitales que Bogotá posee de mapas a fin de aportar a la gestión territorial y de nombrar algunos videojuegos que, desde sus diferentes intereses, aportan a concepciones del desarrollo urbano, se procede a desarrollar el prototipo de City Co.

\footnotetext{
${ }^{15}$ Videojuego de creación, gestión y evolución de ciudades.

${ }^{16}$ Videojuego ubicado en el inicio de la era industrial que cuestiona si se es innovador o explotador de recursos, si se dirige de forma opresiva o libertadora. Recuerda así que el mundo se desarrollará a partir de las normas establecidas.

17 Videojuego en el que se construyen ciudades sobre el mar, pensando en aspectos tales como la movilidad, el acceso a recursos y la generación de energía; se explora para expandir los límites de los dominios creados.

${ }^{18}$ Videojuego que busca concientizar sobre el desarrollo de ciudades con energía sostenible y cuidado medioambiental.

19 Un videojuego en el que se guía a los primeros humanos, se caza, cosecha, se realizan las primeras viviendas, se defiende de otras tribus y se construye edificaciones megalíticas mientras se expande la civilización en el territorio.

${ }^{20}$ Videojuego de construcción, administración y simulación política.

${ }_{21}^{21}$ Videojuego de construcción y gestión territorial.

22 Videojuego de una sociedad desarrollada en un mundo posapocalíptico.

${ }^{23}$ Videojuego que trata de la colonización de Marte y la construcción de una ciudad en ese planeta y la supervivencia en el proceso.

${ }^{24}$ Videojuego sobre una sociedad que debe desarrollarse con base en la energía a vapor debido al eterno invierno.

${ }^{25}$ Simulador de construcción de ciudades.

${ }^{26}$ El desarrollo y la planificación territorial debida a nuestro contexto actual, se caracteriza por las reformas urbanas, ya que solo pensar en expansión no sería una opción por componentes medioambientales y, por tanto, de salubridad de la población.
} 


\subsection{Descripción del videojuego City Co}

Es un videojuego cuyo prototipo se realiza con base en el plan parcial El Triángulo de Bogotá. Algunas de las características de su elección fue la localización muy cercana al centro geográfico de la capital Colombiana, junto con una considerable diversidad de dinámicas urbanas alrededor de este y, así como el hecho de que es uno de los cinco planes parciales gestados en la administración 2016-2019 —periodo en el que se inició City Co—.

City Co maneja los mismos seis ítems de edificación que se plantean en el Plan Parcial El Triángulo — vías, espacio público, vivienda, equipamiento, comercio y oficina-. En esta etapa el jugador se encuentra con la porción del territorio que el plan parcial afecta.

Figura 2. Visualización desde la main camera del videojuego City Co antes de iniciar la propuesta de la reforma urbana.

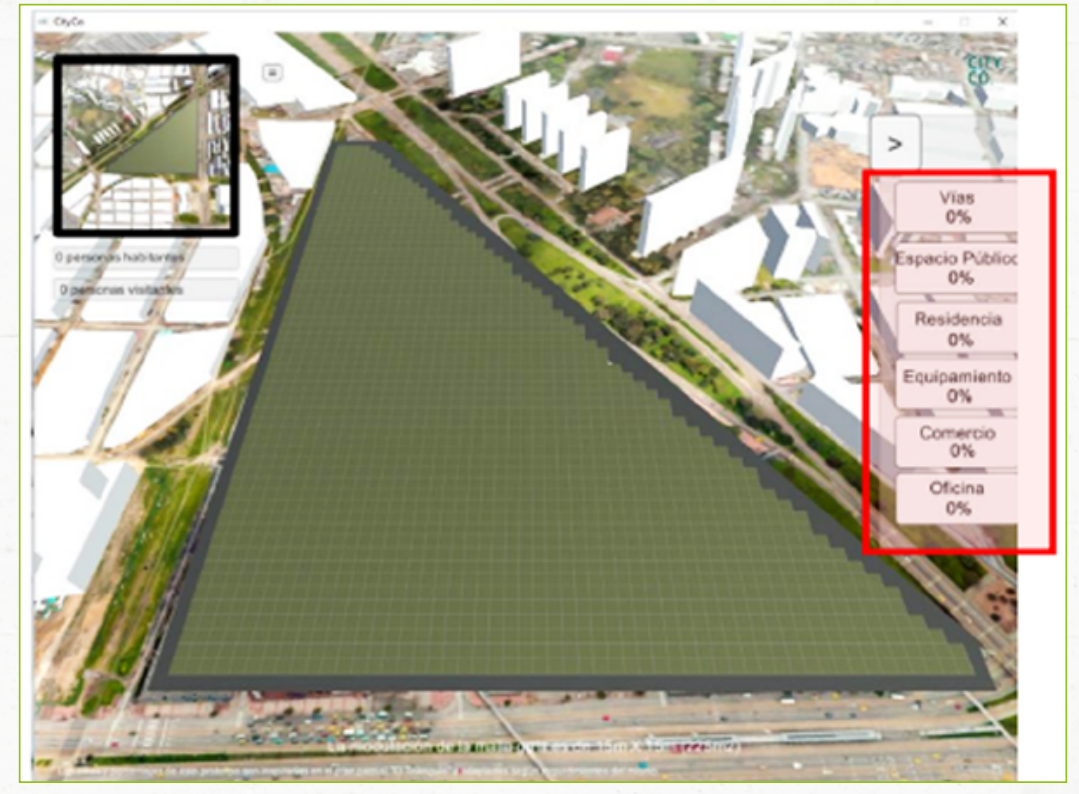

Fuente. Elaboración propia. 
Además de tener la posibilidad de observar estos tipos de construcciones, el jugador puede escoger el tamaño con el que desea realizar su distribución de ordenamiento según el tipo de uso - tal como se muestra en la Figura 3-, y podrá observar cifras de referencia de índices de ocupación con proporciones semejantes a las que maneja el plan parcial, junto con referencias de cantidad demográfica que cada edificación planteada representa. Con base en estos aspectos el usuario podrá hacer su propuesta de ordenamiento territorial para el sector y aprender de forma simultánea algunas de las características que el Gobierno plantea en la reforma urbana de esta porción de la ciudad.

Figura 3. Muestra de ejemplos de escalas de intervención y proyección demográfica del videojuego City Co.

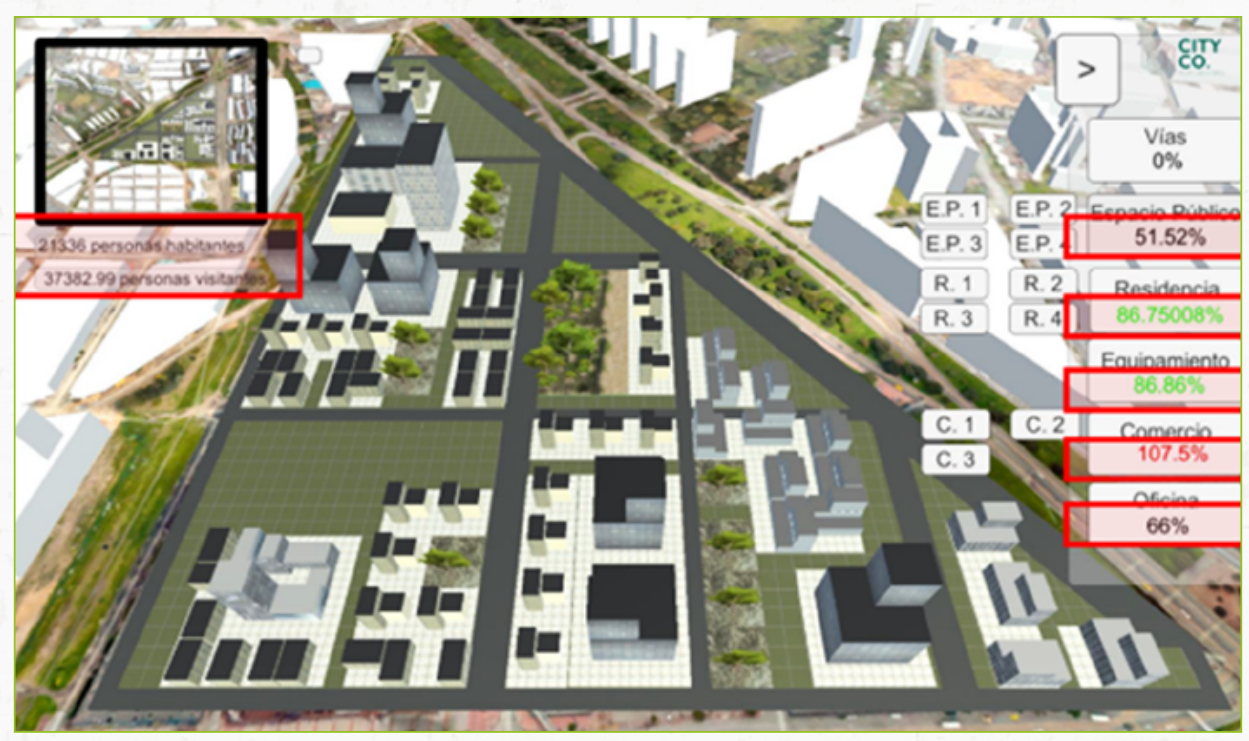

Fuente. Elaboración propia.

El diseño de la volumetría del videojuego se basa en la normativa otorgada en el Plan Parcial El Triángulo. Para la jugabilidad se gamificaron algunas de las pautas sugeridas por Jan Bazant en Manual de criterios de diseño urbano (1984), junto con la aplicación de directrices dadas por Jesse Schell en The art of game design (2008). 
Figura 4. Zoom de relocalización de una edificación de tipo comercial en el videojuego City Co.

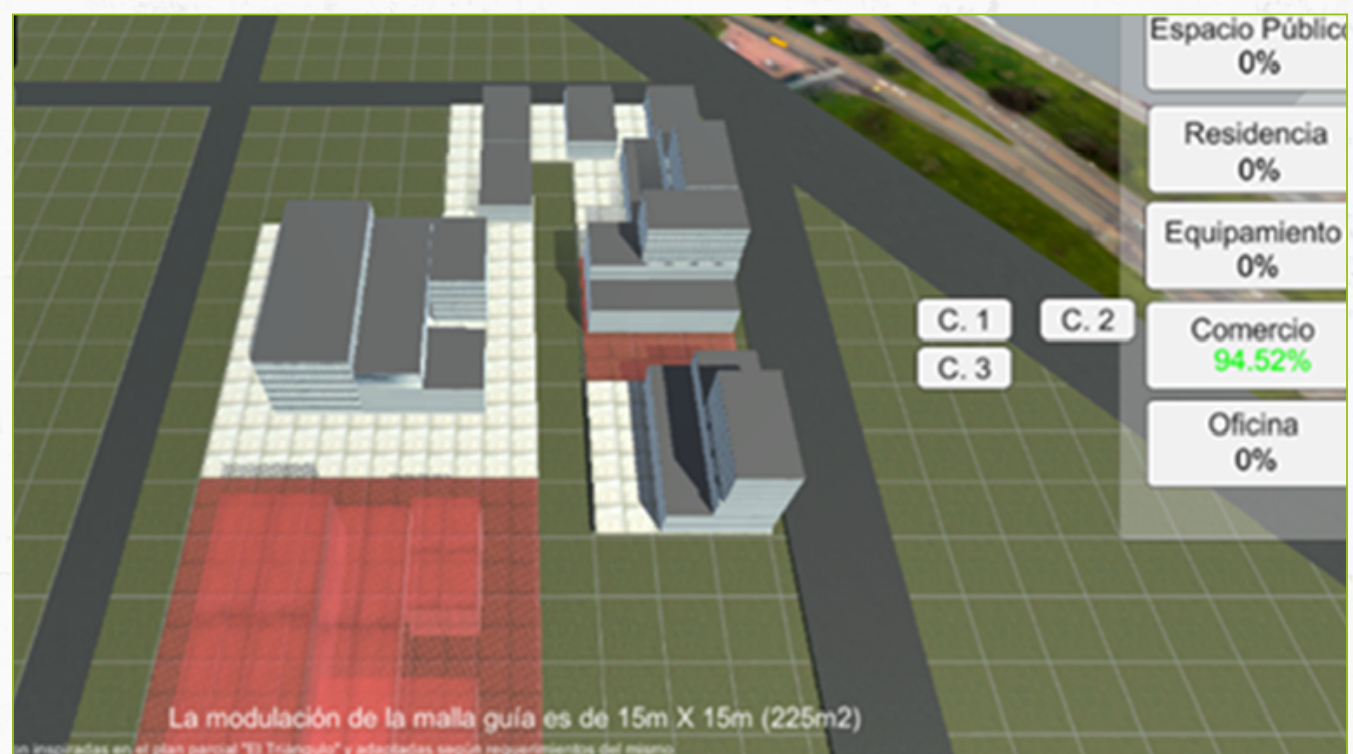

Fuente. Elaboración propia.

Con esto pretendemos no solo dar a conocer la información en cuanto a planteamiento territorial de fragmentos urbanos, sino que buscamos hacerlo de una forma dinámica tridimensional y, junto con esto, brindar la posibilidad de que la comunidad evalúe los planteamientos territoriales existentes y dé su aporte hacia estos. 
Figura 5. Muestra de diferentes cámaras en una muestra de participación ciudadana del videojuego City Co

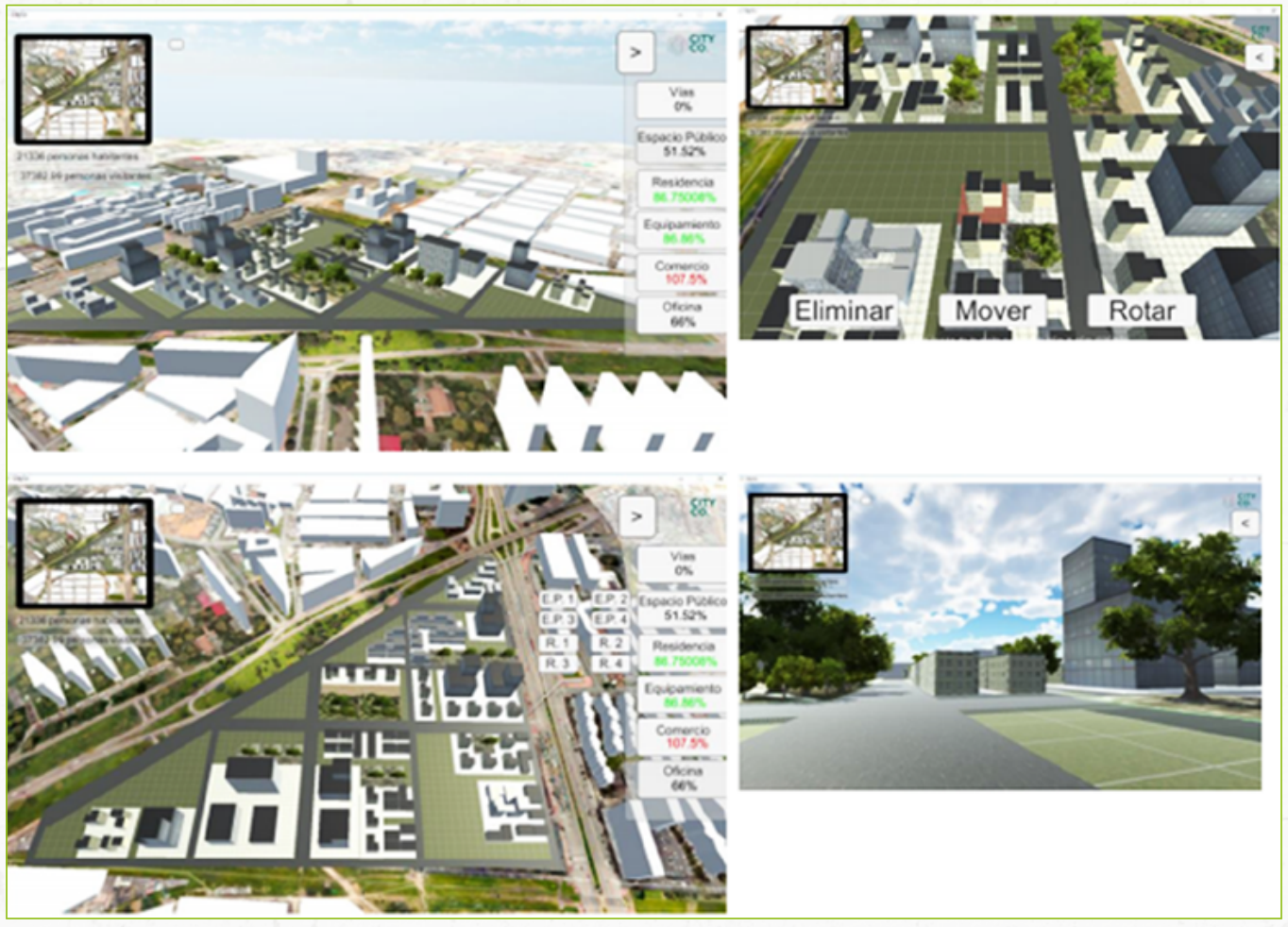

Fuente. Elaboración propia.

\subsubsection{Puesta a prueba}

El proyecto City Co ha tenido participación en universidades en el área de arquitectura y urbanismo. Así, por ejemplo, en la Universidad de los Andes, en su fase preliminar, en la Universidad Piloto de Colombia y en la Universidad de La Salle, entre estudiantes, profesores y participantes de otros lugares en la fase de testeos. También se ha planteado tener cabida en otras universidades y conseguir espacios para dar a conocer el proyecto, sus componentes, los beneficios a la ciudadanía, junto con la proyección, a fin de que más personas puedan participar en la puesta a prueba de estos primeros prototipos. 
Tuvimos participación del 11 al 15 de octubre del 2018 en la feria SOFA, en Corferias, siendo parte del estand de proyectos desarrollados en Unity, con participación de público con interés del proyecto, entre los cuales encontramos aportes tanto técnicos como aportes de proyección social.

Figura 6. Participación ciudadana en un testeo del videojuego City Co.

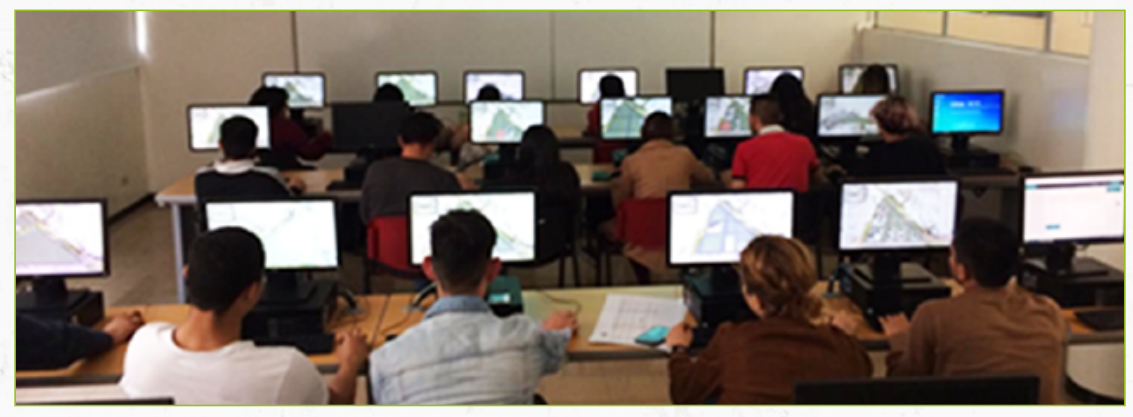

Fuente. Elaboración propia.

Figura 7. Imagen recreada de pensamientos diversos en el momento de la participación ciudadana en un testeo del videojuego City Co.

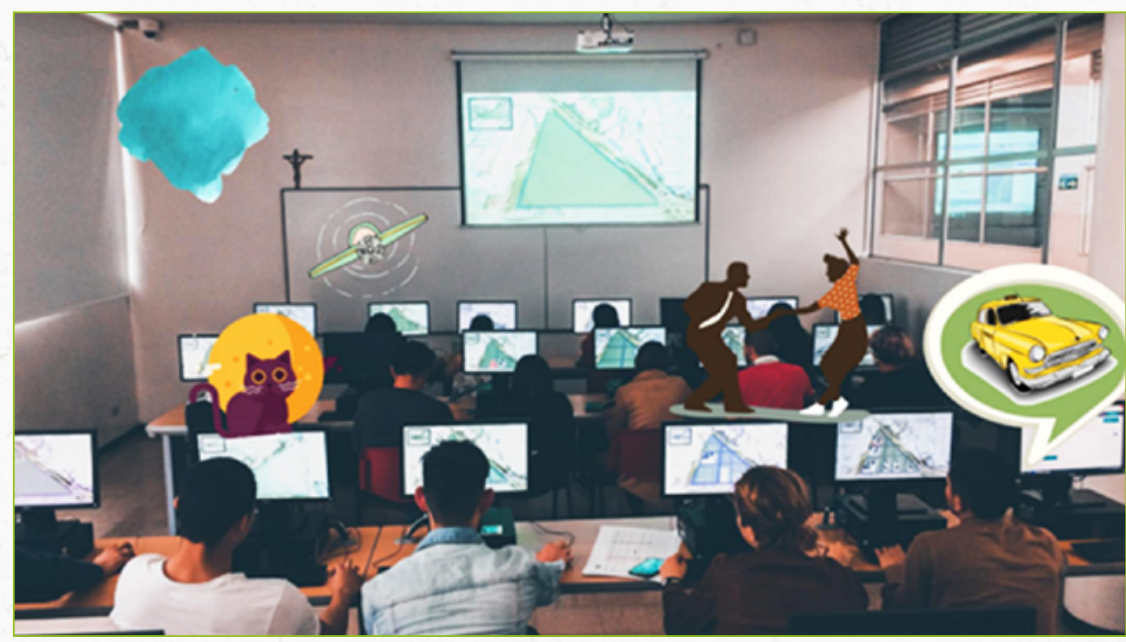

Fuente. Elaboración propia. 


\section{Aprendizajes a partir de CityCo}

En total, participaron en la puesta a prueba de este prototipo de City Co cerca de 400 personas, en su mayoría jóvenes entre 14 y 30 años. El 75 \%, pese a conocer el territorio, desconocía la proyección del plan parcial que ya ha sido aprobado por la Alcaldía, de modo que manifestaron que con el prototipo pudieron acercarse no solo a los usos, a las edificaciones y a las alturas propuestas por el plan parcial, sino a las cifras poblacionales que representan y su implicación en otras áreas de la ciudad. El $12 \%$ corresponde a personas que manejan el tema y han tenido participación en proyectos urbanos, quienes dieron aportes sustanciales al diseño para próximas fases de City Co. El 9 \% son personas que conocían algunas de las proyecciones, bien sea de la Alcaldía o del Plan Parcial, y el $4 \%$ restante son personas extranjeras que observaron el proyecto y manifestaron que tiene potencial de ser aplicado en sus respectivos territorios, con las adecuaciones pertinentes, así como que lo ven útil, potencialmente en cuanto una herramienta que aporta a la regulación de la planificación urbana.

\section{Conclusiones}

Lo expuesto en el transcurso de este trabajo permite concluir, en primera instancia, que los medios tecnológicos que contribuyen a la información y la comunicación del desarrollo territorial en las reformas urbanas aportan a generar interés en el conocimiento y el entendimiento de procesos de planes parciales.

Por otra parte, aunque en los alcances de este trabajo no se implementó la plataforma en interconexión virtual, una constante en pruebas análogas realizadas con una maqueta del videojuego, presentada a modo de juego de mesa, generó mayor interés en el momento de la participación a causa de la interpretación de un rol del jugador. Con esto se comprendió que personificar un área de la planificación urbana y la comunicación entre diferentes entidades ayuda a generar mayor interés $y$, con esto, comprender mejor los procesos en los que se participa.

Un medio tecnológico como un videojuego city builder, emplazado en un entorno real y gamificando procesos de reformas urbanas, aporta a informar de modo significativo a la población joven y promueve en esta inquietudes sobre la planificación territorial. 


\section{Referencias}

Bazant, J. (1984). Manual de criterios de diseño urbano. México: Limusa.

Bogotá, cuarta entre las peores ciudades para vivir en América Latina. (16 de agosto de 2018). El Espectador. Recuperado de https://bit.ly/2WvxZV1

Burden, A. (2014). Cómo los espacios públicos hacen funcionar las ciudades. TED conference. Recuperado de https://bit. ly/3jh10dw

Colomina, B. (2009). Los arquitectos buscamos dioses para adorarlos. Nueva York: La Nación.

Constitución Política de Colombia. (1991). Artículos 20 y 79. Bogotá, Colombia: Norma.

Escallón, C. (2014). Gobernanza en procesos de regeneración urbana, Aproximaciones al caso de Bogotá. Bogotá: Ediciones Uniandes.

Gehl, J. (2011). Life between buildings. Washington: Island Press.

Gehl, J. (2014). Ciudades para la gente. Buenos Aires: Infinito.

Jacobs, J. (2013). Muerte y vida de las grandes ciudades norteamericanas. Madrid: Capitán Swing Libros

Kochen, J. (28 de enero de 2014). Proximidad y asimilación. Arquine. Recuperado de https://www.arquine.com/proximidad-yasimilacion/
Martisi, B. (20 de febrero de 2019). Las 10 mejores «apps» que impulsan la participación ciudadana y la transparencia. Compromiso Empresarial. Recuperado de https://bit. ly/2ONgED3

Mejía, L. (2017). Presentación magistral. Ponencia presentada en el Seminario Desarrollo urbano y participación ciudadana. Universidad de los Andes, Bogotá, 25 de agosto de 2017. Recuperado de https://bit.ly/32tyTW4

Ministerio de Salud y Protección Social. (2018). Sala situacional de las Personas con Discapacidad (PCD). Oficina de Promoción Social. Recuperado de https://bit.ly/2CIL8t1

¿Qué respiran y de dónde proviene la contaminación en Bogotá? (8 de agosto de 2018). Revista Semana. Recuperado de https:// bit.ly/2ZBUU2U

Rojas, C. (2017). Los videojuegos como medio para nuevas estrategias de gestión urbana. Ponencia presentada en el [2017] Congreso Internacional de Cultura Visual. Universidad CEU Abat Oliba, Barcelona, España, 22-23 de mayo.

Schell, J. (2008). The art of game design. A book of lenses. EE. UU.: Morgan Kaufmann. DOI: https://doi. org/10.1201/9780080919171

Vega, J. P. (8 de junio de 2017). Bogotá tiene un déficit de 74\% en espacio público. La República. Recuperado de https://bit. ly/32urxSg 